\title{
25 Research Square \\ Insights Into the Architecture of Human-induced Polygenic Selection in Duroc Pigs
}

\section{Zitao Chen}

Zhejiang University

Jinyan Teng

South China Agricultural University

Shuqi Diao

South China Agricultural University

\section{Zhiting Xu}

South China Agricultural University

Shaopan Ye

Shantou University

\section{Dingjie Qiu}

Fujian Yongcheng Agricultural \& Animal Husbandry Sci-Tech Group Co., Ltd.

\section{Zhe Zhang}

Zhejiang University

\section{Yuchun Pan}

Zhejiang University

Jiaqi Li

South China Agricultural University

\section{Qin Zhang}

Shandong Agricultural University

Zhe Zhang ( $\square$ zhezhang@scau.edu.cn )

South China Agricultural University https://orcid.org/0000-0001-7338-7718

\section{Research Article}

Keywords: Artificial selection, Pig, Selection signatures, GWAS, Reproductive organ

Posted Date: March 7th, 2022

DOI: https://doi.org/10.21203/rs.3.rs-1388066/v1

License: (1) This work is licensed under a Creative Commons Attribution 4.0 International License. Read Full License 


\section{Abstract \\ Background}

As one of the most utilized commercial composite boar lines, Duroc pigs have been introduced to China and undergone strongly human-induced selection over the past decades. However, the efficiencies and limitations of previous breeding of Chinese Duroc pigs are largely understudied. The objective of this study was to uncover directional polygenic selection in the Duroc pig genome, and investigate points overlooked in the past breeding process.

\section{Results}

Here, we utilized the Generation Proxy Selection Mapping (GPSM) on a dataset of 1,067 Duroc pigs with $8,766,074$ imputed SNPs. GPSM detected a total of 5,649 putative SNPs actively under selection in the Chinese Duroc pig population, and the potential functions of the selection regions were mainly related to production, meat and carcass traits. Meanwhile, we observed that the allele frequency of variants related to teat number (NT) relevant traits was also changed, which might be influenced by genes that had pleiotropic effects. First, we identified the direction of selection on NT traits by $\hat{G}$, and further pinpointed large-effect genomic regions associated with NT relevant traits by selection signature and GWAS. Combining results of NT relevant traitsspecific selection signatures and GWAS, we found three common genome regions, which were overlapped with QTLs related to production, meat and carcass traits besides "Teat number" QTLs. This implied that there were some pleiotropic variants underlying NT and economic traits. We further found that rs346331089 has pleiotropic effects on NT and economic traits, e.g., litter size at weaning (LSW), litter weight at weaning (LWW), days to $100 \mathrm{~kg}$ (D100), backfat thickness at $100 \mathrm{~kg}$ (B100), and loin muscle area at $100 \mathrm{~kg}$ (L100) traits.

\section{Conclusions}

The selected loci that we identified across methods displayed the past breeding process of Chinese Duroc pigs, and our findings could be used to inform future breeding decision.

\section{Introduction}

About 9, 000 years ago, pigs were domesticated in multiple locations around the world.

And then, high-intensity artificial selection has been applied to the genetic improvement of agriculturally important traits [1]. As selection at favorable mutations have played an essential role in the domestication and genetic improvement of animals, the frequency of favorable mutations will increase rapidly and this process is called selective sweep. Different approaches have been proposed for the identification of selective sweep, e.g., the genetic diversity ratio $\left(\theta_{\pi}\right)$ and Wright's fixation index $\left(F_{\mathrm{ST}}\right)$ [2]. Most approaches are designed to identify genomic regions with large-effect and have successfully identified large-effect quantitative trait loci (QTL) under selection that controls pig traits, e.g., coat color, meat quality, and fertility [3]. Indeed, agriculturally important traits are usually controlled by many mutations of small effect. Recently, some methods have been 
developed to detect polygenic selection, e.g., the Generation Proxy Selection Mapping (GPSM) allows us to observe how complex polygenic selection alters the genome over short timescales in a trait-agnostic manner [4] and $\hat{G}$ can be used to powerfully identify selection on highly polygenic traits [5].

Duroc pig, as an older breed of domestic pig, was developed in America and formed after a long period of artificial selection. In modern pig industry, Duroc pig is one of the most utilized commercial composite boar lines, and well-known for its growth, feed conversion efficiency, carcass and meat quality traits [6]. Because pleiotropic function exerts, high-intensity artificial selection on production traits potentially causes the weakening of other traits. For instance, the average teat number of Duroc pig breed was lower than that of the Large White [7] and Landrace pig breeds [8].

Herein, we first used two methods (GPSM and $\hat{G}$ ) to detect polygenic selection in a factory-farmed Duroc pigs. Then, we performed genome-wide association studies (GWAS) and selection-mapping protocols $\left(F_{\mathrm{ST}}\right.$ and $\theta_{\pi}$ ratio) to identify the potential large-effect NT trait-related genomic regions. Further, we conducted a comprehensive analysis to identify the putative pleiotropic genomic regions. The results of this study uncover the genetic improvement of Chinese Duroc pig population over the past decade and will be used to inform future breeding decision.

\section{Materials And Methods Ethical statement}

All experiments in this study were approved by the Animal Care Committee of South China Agricultural University (Guangzhou, People's Republic of China) with approval number SCAU\#2013-10, and the experiments were performed according to the regulations and guidelines established by this committee.

\section{Sample preparation and sequencing}

A total of 1,067 animals consisted of 984 females and 83 males from a Duroc pig population managed in Fujian, China, were used in this study. These animals were born between 2009 and 2017. All phenotypic records were extracted from the Herdsman swine management platform (S\&S Programming, Lafayette, IN, USA). The number of left teats, right teats were recorded by simple counting. The number of teats was the sum of the teat number at both sides. In addition, the number of left teats, right teats, and teats of each individual was counted at birth and the malformed teats were not recorded. Furthermore, we obtained the phenotypic ( litter size at weaning (LSW), litter weight at weaning (LWW), days to $100 \mathrm{~kg}$ (D100), backfat thickness at 100 $\mathrm{kg}$ (B100), and loin muscle area at $100 \mathrm{~kg}($ L100)) data from our previous studies $[9,10]$.

In this study, we extracted genomic DNA from the ear tissue of 1067 Duroc pigs using the TaKaRa MiniBEST Universal Genomic DNA Extraction Kit (Version 4.0), then checked using agarose gel electrophoresis and quantified with a NanoDrop 2000 (Thermo Scientific, Waltham, MA, USA). Either the Illumina PorcineSNP60 BeadChip (Illumina, San Diego, CA, USA) comprising 63, 480 SNPs or the GeneSeek GGP-Porcine chip (Neogen Corporation, Lansing, MI, USA) comprising 51, 558 SNPs were used to genotype the individuals. The common SNPs contained 33, 359 SNPs between two chips were retained. Among the Duroc pigs used in the current 
study, 50 key individuals were selected according to maximize the expected genetic relationships as in [11]. These individuals were re-sequenced with 150 bp paired-end reads using the Illumina HiSeq 3000 platform. In the raw reads, the adaptor polluted reads and multiple $\mathrm{N}$ reads (where $\mathrm{N}>10 \%$ of one read) were removed using Trim Galore version 0.6.1 (http://www.bioinformatics.babraham.ac.uk/projects/trim_galore/) to produce the clean reads. Further, the clean data were aligned to the Sus scrofa 11.1 reference genome using BurrowsWheeler Aligner (BWA) version 0.7.15 [12]. The genome analysis toolkit GATK version 4.1.2.0 [13] was used to detect the SNPs using a Bayesian model, a total of 19, 754, 293 SNPs was found. Subsequently, genotype imputation was performed, treating 50 key individuals with sequencing data as reference panel, and the remaining 1017 individuals with SNP arrays data were imputed to whole genome sequence (WGS) data using Beagle version 5.0 [14]. Quality control of SNPs was implemented using VCFtools version 0.1 .14 [15] with a $\mathrm{DR}^{2}$ of $0.8, \mathrm{MAF}$ of $1 \%$, missing rate of $20 \%$, HWE of 0.00001 , and sites with a mean depth greater than or equal to 3. Then, PLINK version 1.09 [16] was utilized to convert file formats of the independent SNPs. Hence, a total of 1067 individuals with 8, 766, 074 SNPs were eligible for inclusion in the following analyses (Figure S1).

\section{Detecting of polygenic selection}

An animal's age as of December 21, 2017 was used as the generation proxy in GPSM, and we fit a univariate genome-wide linear mixed model as follows:

$$
\mathbf{Y}=\mathbf{X} \boldsymbol{\beta}+\mathbf{Z u}+\mathbf{e}
$$

Where $\mathbf{Y}$ is an individual's generation proxy; $\boldsymbol{\beta}$ is the estimated effect size for each $\mathrm{SNP}_{\mathrm{s}} ; \mathbf{u}$ is polygenic term and is set as $\mathbf{u} \sim \mathbf{N}\left(\mathbf{0}, \mathbf{G} \boldsymbol{\sigma}_{a}^{2}\right) . \mathbf{X}$ and $\mathbf{Z}$ are incidence matrices for $\boldsymbol{\beta}$ and $\mathbf{u} ; \mathbf{e}$ is the random residuals and is set as $\mathbf{e} \sim \mathbf{N}\left(\mathbf{0}, \mathbf{I} \sigma_{e}^{2}\right)$, where $\mathbf{I}$ is an identity matrix. We used FDR corrected q-values to control for multiple-testing and SNPs with $\mathrm{q}$-value $<0.1$ was deemed as significant variants [4].

We estimated a composite statistic ${ }^{\hat{G}}$ on left teats, right teats, and total teats traits to test for the direction of selection of NT relevant traits. We fit a ridge regression best linear unbiased prediction (RRBLUP) model with NT traits as the response and the year and season of individuals at birth as fixed effects.

\section{Selection-mapping protocols}

The pairwise difference between each year were tested using a Welch Two Sample t-test and listed in Table S1. There were significant differences in the number of teats between sows born on 2009-2011 and born after 2011. To further detect NT relevant traits-specific selection signatures, a phenotypic differential population pair, (1) larger teat number (LT) group: 45 sows born before 2011 with larger number of teats $(14.16 \pm 0.01)$ and (2) smaller teat number (ST) group: 45 sows born on 2017 with smaller number of teats $(12.00 \pm 0.00)$, 
was created. The $\theta_{\pi}$ ratio and $F_{\mathrm{ST}}$ were used to detect signatures of selection in this phenotypic differential population pair with the use of a sliding window method ( $50 \mathrm{~kb}$ window and $10 \mathrm{~kb}$ step). The $\theta_{\pi}$ ratio between LT group and ST group was calculated as $\ln \left(\theta_{\pi \mid L T} / \theta_{\pi \mid S T}\right)$. In addition, the $1 \%$ of windows with the highest $\theta_{\pi}$ ratio and $F_{\mathrm{ST}}$ values was considered the potential selection regions.

\section{Association analyses}

GWAS was performed using a mixed linear model, as follows:

$$
\mathbf{Y}=\mathbf{W} \boldsymbol{\alpha}+\mathbf{X} \boldsymbol{\beta}+\mathbf{Z u}+\mathbf{e}
$$

where $\mathbf{Y}$ is the number of left teats, right teats, and total teats of the individuals; $\boldsymbol{\alpha}$ is a vector of fixed effect, including the year and season of individuals at birth; $\boldsymbol{\beta}$ is the substitution effect of the $\mathrm{SNP}_{5} ; \mathbf{u}$ is the random effect and is set as $\mathbf{u} \sim \mathbf{N}\left(\mathbf{0}, \mathbf{G} \boldsymbol{\sigma}_{a}^{2}\right)$, where $\mathbf{G}$ is the genomic relationship matrix; $\mathbf{W}, \mathbf{X}$, and $\mathbf{Z}$ are incidence matrices for $\boldsymbol{\alpha}$, $\boldsymbol{\beta}$, and $\mathbf{u} ; \mathbf{e}$ is the random residuals and is set as $\mathbf{e} \sim \mathbf{N}\left(\mathbf{0}, \mathbf{I} \sigma_{e}^{2}\right)$, where $\mathbf{I}$ is an identity matrix. The analyses were performed using GEMMA software [17]. The Bonferroni correction was applied to filter the potential SNPs: SNPs with permuted $P$-values lower or equal than $0.05 / \mathrm{N}$ (N is the number of the independent markers defined as a set of SNPs with pairwise $r$ square value higher than 0.4 ), were regarded as genome-wide significant SNPs. SNPs with a $P$-value higher than $0.05 / \mathrm{N}$ but lower than $1 / \mathrm{N}$ were considered as genome-wide suggestive significant SNPs. Then, quantile-quantile (Q-Q) plots were drawn and the inflation factors $(\lambda)$ were calculated to check the population stratification.

In order to uncover pleiotropic effects of the intron mutation (rs346331089) and its potential effect on the phenotype in Duroc pigs, we utilized our previous reported data sets of economic traits, followed by implementing the association analyses between rs346331089 and economic traits using PLINK with "-linear" parameter.

\section{Tissue-specific genes and pCADD scores annotation}

We downloaded gene expression matrix of Duroc pig tissues (i.e., fat, heart, liver, muscle, spleen, cerebellum, cerebrum, duodenum, kidney, lung, thymus) from publicly available datasets [18]. As in Zhao et al. (2021), the expression of genes in a given tissue with at least three times higher than that in other tissues were classified 
as tissue-specific genes. Meanwhile, we selected human-pig homologous genes from Ensembl release 105 (http://asia.ensembl.org/index.html) and used the human protein atlas [19] to further explore the expression of human-pig homologous genes with high confidence. Furthermore, PCADD scores were retrieved from public databases [20] to prioritize variants.

\section{Functional enrichment analysis}

The Animal QTL Database [21] was used to annotate the potential functions of the selection regions. QTL enrichment analyses based on a bootstrap simulation for each QTL were conducted to annotate selection signatures, and the adjusted $P$-value based on multiple tests less than 0.05 were retained. Furthermore, the genes located in putative selection regions were identified using R package GALLO [22]. Then, the positional candidate genes overlapped with the genomic regions for NT traits were extracted based on Sus scrofa 11.1 reference genome assembly. We used $\mathrm{R}$ package clusterProfiler [23] to conduct enrichment analyses, then the Kyoto Encyclopedia of Genes and Genomes (KEGG) pathways and GO terms with Benjamini-Hochberg method adjusted $P$-value $<0.05$ were selected.

\section{Results}

\section{Whole-genome sequencing and imputation}

The genomic DNA extracted from key individuals $(N=50)$ of a factory-farmed Duroc pig population was resequenced with 150 bp paired-end reads using the Illumina HiSeq 3000 platform. A total of 19, 754, 293 SNPs was found, of which $20.14 \%$ were novel in comparison with the latest pig SNP database (https://ftp.ncbi.nih.gov/snp/organisms/archive/pig_9823/chr_rpts/). In addition, transition-to-transversion (TS/TV) ratios of SNPs was 2.33. With the use of Variant Effect Predictor (VEP, http://asia.ensembl.org/info/docs/tools/vep/index.html) to variant functional annotation, we obtained location of the variants and the most severe consequence of your variants on the protein sequence. The most SNPs (59.55\% of all SNPs) were located in intron regions, followed by intergenic (19.94\%), downstream genic $(8.71 \%)$, and upstream genic regions (8.37\%). Moreover, the plot showed that SNPs were evenly distributed across porcine autosomes (Figure S1).

After imputation with key individuals as reference panel, we obtained a large-scale genotyping dataset contained 8,766,074 high-quality SNPs with dosage R-squared $\left(D R^{2}\right)>0.8$. The proportion of each consequence types was broadly similar with reference panel, and the distribution of SNPs was shown in Figure S2. Therefore, a total of 1067 Duroc pigs with $8,766,074$ SNPs available for further analyses.

\section{Identification of human-induced polygenic selection}

Based on results from the GPSM analyses (Figure 1A), 5,649 SNPs (q-values < 0.1) were significantly associated with birth date in the Chinese Duroc pig population (Table S2). We explored the potentially biological function of detected GPSM signals with genome annotation, e.g., tissue-specific genes and publicly available QTLs. 
22 out of 24 tissue-specific genes covered by GPSM signals were homologous between pig and human with high confidence, and a majority of these genes had similar expression patterns with tissue specificity in human (Table 1). Further, we found that the expression levels of cerebellum, cerebrum, and fat tissue-specific genes covered by GPSM signals were the most conserved between pig and human. The strongest association signals located in Sus scrofa chromosome (SSC) 3 (Figure 1B) and 8 (Figure 1C), we observed high LD between the "lead" SNP and SNPs around the "lead" SNP. QTL enrichment analyses with the signals showed that production, meat and carcass traits were mostly significantly enriched (Figure 1D). Interestingly, we noticed that "Teat number" QTL was also significantly enriched.

To further estimate the direction of selection on NT relevant traits, we summarized the phenotypic records and found that there were significant differences in the number of teats between pigs born on 2009 and 2017 (a Welch Two Sample t-test $P$-value $=0.0039)$. Moreover, we conducted $\hat{G}$ analyses with phenotypic records, and observed significant evidence of selection for increased left teat $(P$-value $=0.0003$, Figure $2 A)$, right teat $(P$ value $=0.0009$, Figure 2B), and total teat number $(P$-value $=0.0002$, Figure 2C). These results uncovered that NT relevant traits had been sufficiently human-induced selected in the past few years in the Chinese Duroc pig population.

\section{Teat number relevant traits-specific selection signature detection}

We conducted detection of traits-specific selection signatures based on the constructed population pairs with extreme differences in total teat number. Here, we detected 401 positive selection signatures (Figure 2D), of which 209 loci reflected the loss of nucleotide diversity in LT group relative to ST group, while 192 loci were opposite. QTLs enrichment analyses showed that "Teat number" QTL was indeed enriched, meanwhile, we noticed that the selection signatures significantly overlapped with several QTLs related to production, meat and carcass traits.

In total, 13 out of 78 protein coding genes were covered by these NT relevant traits-specific selection signatures, and showed tissue-specific expression levels. Thereinto, ASPH, CCDC85C, CYP46A1, ASGR2, PNLIP, PNLIPRP1, DPP10, EPHA4, and PLCB4 had similar RNA tissue specificity in pig and human (Table 2 and S3). Functional annotations were significantly enriched in lipid metabolism related pathways and terms, e.g., "pancreatic secretion", "fat digestion and absorption", "glycerolipid metabolism", and "lipid catabolic process". These results proved that the trait-specific selection signatures were mainly caused by phenotypic difference and uncovered that NT relevant traits-related candidate genes played potential roles in lipid metabolism.

\section{Imputed Sequence-based GWAS for teat number relevant traits}

Using the number of left teats, right teats, and total teats as phenotypic records, a total of 1313 putative loci were significantly associated with NT relevant traits, thereinto, 4, 71, and 164 putative loci were uniquely detected in imputed sequence-based GWAS for the number of left teats, right teats, and total teats, respectively. Meanwhile, 773 of all putative loci were shared by GWAS for these three NT relevant traits (Figure S3). SPATA6, 
VRTN, FOXN3, KCNK10, RND3, and RIF1 were covered and identified as the promising candidate underlying NT relevant traits. We found that some of the promising candidate genes were also associated with fat-related traits, e.g., KCNK10 and RND3, which was consistent with the results of QTL enrichment analyses (Figure S4).

\section{Comparison between traits-specific selection signature and GWAS}

Combining results of NT relevant traits-specific selection signatures and GWAS, we found three common genome regions, located in SSC 6, 7, and 15, respectively. In these regions, rs346331089 (Figure 4), rs322980623 (Figure S5), and rs324534752 (Figure S6) got the highest pCADD score. The regions located in SSC6 significantly enriched in the QTLs related to litter size, rump width, oleic acid content, and top line conformation traits (Figure S7A), the regions located in SSC7 were associated with obesity index, head weight, mean corpuscular volume, mean corpuscular hemoglobin concentration, and cannon bone circumference traits (Figure S7B), and the regions located in SSC 15 overlapped with QTLs related to backfat between 3nd and 4th last ribs and hematocrit traits (Figure S7C). These results suggested that there were a few pleiotropic genes in these genome regions, influencing both NT and economic traits.

\section{Identification of pleiotropic variants underlying teat number and economic traits}

As one of the most utilized commercial composite boar lines, it is well known that Duroc pigs had been under selection for production and meat quality traits. Nevertheless, as mentioned above, NT relevant traits have experienced human-induced selection in the Chinese Duroc pig population. We assumed that a few pleiotropic variants were putative contradictive loci that played an opposite directional role in teat number and economic traits, leading to decreased left teat, right teat, and total teat number.

We further found that rs346331089 has pleiotropic gene action on NT and economic traits, e.g., LSW, LWW, D100, B100, and L100 traits (Figure 4). Thereinto, genotype effects of rs346331089 on NT traits showed similar trends in its effects on D100 and B100 traits, whereas its effects had opposite patterns for LSW, LWW, and L100 traits. Overall, human-induced selection for the genetic improvement (e.g., faster growth rates, thinner backfat thickness, and larger loin muscle area) at the genome regions might lead to the decreasing number of teats.

\section{Discussion}

In this study, we detected polygenic selection in a factory-farmed Duroc pigs. Genes related to teat relevant traits were then dissected by combined analyses of selection signatures and GWAS. Pleiotropic variants underlying teat number and economic traits were further confirmed.

\section{Human-induced polygenic selection in Duroc pigs}


Based on the results of GPSM analyses, we infer that the Chinese Duroc pig population was mainly subjected to high-intensity artificial selection on production and meat quality traits, in line with the breeding goals of Duroc pigs [24]. Further gene annotations showed a set of candidate genes involved in artificial selection, e.g., GRIK2, JAKMIP1, GRID2, FASN, GAS7, SELENOP, and SGCZ. GRIK2 belongs to the kainate family of glutamate receptors. Previous study suggested that GRIK2 played a role in affecting intermuscular fat level [25]. We observed the frequencies of GRIK2 mutations were obviously different between the population in 2009 and 2017, these mutations might affect the normal transcription and expression of GRIK2 and further have impacts on intermuscular fat level, which is an important meat quality parameter. Also, JAKMIP1 involved in the actions of neurons, which are central regulators in maintaining the balance between food intake and energy expenditure, and further regulated fat deposition in muscle [26]. FASN is related to lipogenesis and has been found the potential roles in the determination of feed conversion and meat color in pigs [27]. GAS7 was implicated in influencing the fatty acid composition in pigs [28]. Moreover, SGCZ has potential functions in lipid metabolism [29]. In addition, a few genes (e.g., GRID2 and SELENOP) were briefly reported to be associated with reproduction traits [30,31]. Likewise, we observed that the GPSM signals mostly significantly enriched in production, meat and carcass traits, as other traits were rarely enriched. Altogether, genetic improvement of Duroc pigs in China through selection on genes that are correlated with economic characters (e.g., production and meat quality) has been mainly considered during artificial selection.

\section{Traits-specific selection signature and GWAS for teat number relevant traits}

During the rapid improvement of the performance of economic traits, the number of teats has decreased in the Chinese Duroc pig population. We hypothesized that genetic correlations between NT and economic traits.

Traits-specific selection signatures were high enriched in both teat number relevant traits and fat-related traits, which confirmed that the hypothesis was correct. Moreover, several candidate genes (e.g., ASPH, CYP46A1, PNLIP, PNLIPRP1, DPP10, EPHA4, and PLCB4) overlapped with traits-specific selection signatures have underlying correlations with economically significant traits. $A S P H$ are involved with tissue morphology, skeletal and muscle development, and fat deposition [32]. Also, CYP46A1, PNLIP, and DPP10 have been identified as regulators of lipid metabolism [33-35]. EPHA4, which was detected in the endometrium during embryo implantation in pigs, was found to have relationships with reproduction traits [36]. Moreover, PLCB4 was implicated in growth and stature traits, and has been identified as genes under directional selection between Duroc and Duroc synthetic pig populations [37]. Further, we focused on detection of candidate genes that related to NT relevant traits. Interestingly, several genes located on the region ranged from $110.13 \mathrm{Mb}$ to 110.47 Mb on chromosome 7 were found, and also reported in previous GWAS for NT trait in pigs [38]. Among these positional candidate genes, $K C N K 10$, as a member of tandem pore domain potassium channel family, involves in stabilizing the negative resting membrane potential and counterbalancing depolarization. KCNK10 has been reported that it is a regulator of mitotic clonal expansion during the adipocyte differentiation [39]. FOXN3 could be considered as a candidate gene for exterior traits in pigs [40]. In addition, RND3 was identified as candidate genes for residual feed intake in pigs [41] and RIF1 was one of putative regulatory factors that contribute to the molecular mechanisms that underlie fat content and energy balance in muscle [42]. Overall, 


\section{Pleiotropic variants underlying teat number and economic traits}

Further, we found that genotype effects of rs346331089 on NT reflected similar trends with D100 and B100 traits, whereas had opposite trends with LSW, LWW, and L100 traits. The variant rs346331089 located in the intron of SPATA6, which is one of sperm-specific genes. Previous studies suggested the regulation of the expression pattern of SPATA6 linked to spermatogenesis in Hu sheep [43] and inactivation of SPATA6 leaded to acephalic spermatozoa and male sterility in mice [44]. These results above manifested high-intensity directional selection on certain economic traits might influenced the number of teats in pigs.

\section{Conclusions}

In this study, we detected polygenic selection in a factory-farmed Duroc pigs and dissected the candidate genes related to teat number relevant traits by combined analyses of selection signatures and GWAS. The variant rs346331089 has pleiotropic effects on teat number relevant traits and economic traits. Our findings showed that genetic improvement through human-induced selection on genes that are correlated with economically important traits may lead to the decreasing number of teats, and contributed to guide the further breeding of Duroc pigs.

\section{Abbreviations}

GPSM: Generation Proxy Selection Mapping; NT: Teat number; LSW: Litter size at weaning; LWW: Litter weight at weaning; D100: Days to $100 \mathrm{~kg}$; B100: Backfat thickness at $100 \mathrm{~kg}$; L100: Loin muscle area at $100 \mathrm{~kg}$; QTL: Quantitative trait loci; GWAS: Genome-wide association study; G: Standardized relatedness matrix; LD: Linkage disequilibrium; MAF: Minor allele frequency; Omics: multiple genomelevel; SNP: Single nucleotide polymorphism; WGS: Whole genome sequencing;

\section{Declarations}

\section{Ethics approval and consent to participate}

Not applicable

\section{Consent for publication}

Not applicable

\section{Availability of data and materials}


The datasets used and analyzed during this study are available from the corresponding author upon reasonable request.

\section{Competing interests}

The authors claim that there are no conflicts of interest.

\section{Funding}

This work was financially supported by China Agriculture Research System of MOF and MARA and the National Natural Science Foundation of China (32022078).

\section{Authors' contributions}

Data curation: ZZ¹ , JQL, and ZTX; Funding acquisition: ZZ1 , JQL; project administration: ZTC; software: ZTC, JYT, and SQD; writing-original draft: ZTC, ZZ1 ; writing-review and editing: SPY, ZZ², YCP, and QZ.

\section{Acknowledgements}

We would like to thank Fujian Yongcheng farming and animal husbandry Co., Ltd for providing the data and offering the opportunity to conduct this study.

\section{References}

1. Bosi P, Russo V. The production of the heavy pig for high quality processed products. Ital J Anim Sci. 2004;3:309-21.

2. Weir BS, Cockerham CC. Estimating F-Statistics for the Analysis of Population Structure. Evolution (N Y). 1984;38:1358.

3. Diao S, Huang S, Chen Z, Teng J, Ma Y, Yuan X, et al. Genome-Wide Signatures of Selection Detection in Three South China Indigenous Pigs. Genes (Basel). 2019;10:346.

4. Rowan TN, Durbin HJ, Seabury CM, Schnabel RD, Decker JE. Powerful detection of polygenic selection and evidence of environmental adaptation in US beef cattle. Buerkle A, editor. PLOS Genet. 2021;17:e1009652.

5. Beissinger T, Kruppa J, Cavero D, Ha N-T, Erbe M, Simianer H. A Simple Test Identifies Selection on Complex Traits. Genetics. 2018;209:321-33.

6. Diao S, Luo Y, Ma Y, Deng X, He Y, Gao N, et al. Genome-wide detection of selective signatures in a Duroc pig population. J Integr Agric. 2018;17:2528-35.

7. Duijvesteijn N, Veltmaat JM, Knol EF, Harlizius B. High-resolution association mapping of number of teats in pigs reveals regions controlling vertebral development. BMC Genomics. 2014;15:542. 
8. Lopes MS, Bastiaansen JWM, Harlizius B, Knol EF, Bovenhuis H. A Genome-Wide Association Study Reveals Dominance Effects on Number of Teats in Pigs. Moore S, editor. PLoS One. 2014;9:e105867.

9. Zhang, Chen, Ye, He, Huang, Yuan, et al. Genome-Wide Association Study for Reproductive Traits in a Duroc Pig Population. Animals. 2019;9:732.

10. Zhang Z, Chen Z, Diao S, Ye S, Wang J, Gao N, et al. Identifying the complex genetic architecture of growth and fatness traits in a Duroc pig population. J Integr Agric. 2021;20:1607-14.

11. Ye S, Yuan X, Lin X, Gao N, Luo Y, Chen Z, et al. Imputation from SNP chip to sequence: a case study in a Chinese indigenous chicken population. J Anim Sci Biotechnol. 2018;9:30.

12. Li H, Durbin R. Fast and accurate long-read alignment with Burrows-Wheeler transform. Bioinformatics. 2010;26:589-95.

13. McKenna A, Hanna M, Banks E, Sivachenko A, Cibulskis K, Kernytsky A, et al. The Genome Analysis Toolkit: A MapReduce framework for analyzing next-generation DNA sequencing data. Genome Res. 2010;20:1297-303.

14. Browning BL, Zhou Y, Browning SR. A One-Penny Imputed Genome from Next-Generation Reference Panels. Am J Hum Genet. 2018;103:338-48.

15. Danecek P, Auton A, Abecasis G, Albers CA, Banks E, DePristo MA, et al. The variant call format and VCFtools. Bioinformatics. 2011;27:2156-8.

16. Purcell S, Neale B, Todd-Brown K, Thomas L, Ferreira MAR, Bender D, et al. PLINK: A Tool Set for WholeGenome Association and Population-Based Linkage Analyses. Am J Hum Genet. 2007;81:559-75.

17. Zhou X, Stephens M. Genome-wide efficient mixed-model analysis for association studies. Nat Genet. 2012;44:821-4.

18. Zhao Y, Hou Y, Xu Y, Luan Y, Zhou H, Qi X, et al. A compendium and comparative epigenomics analysis of cis-regulatory elements in the pig genome. Nat Commun. 2021;12:2217.

19. Uhlén M, Fagerberg L, Hallström BM, Lindskog C, Oksvold P, Mardinoglu A, et al. Tissue-based map of the human proteome. Science (80- ). 2015;347.

20. Groß C, Derks M, Megens H-J, Bosse M, Groenen MAM, Reinders M, et al. pCADD: SNV prioritisation in Sus scrofa. Genet Sel Evol. 2020;52:4.

21. Hu Z-L, Park CA, Reecy JM. Building a livestock genetic and genomic information knowledgebase through integrative developments of Animal QTLdb and CorrDB. Nucleic Acids Res. 2019;47:D701-10.

22. Fonseca PAS, Suárez-Vega A, Marras G, Cánovas Á. GALLO: An R package for genomic annotation and integration of multiple data sources in livestock for positional candidate loci. Gigascience. 2020;9.

23. Yu G, Wang L-G, Han Y, He Q-Y. clusterProfiler: an R Package for Comparing Biological Themes Among Gene Clusters. Omi A J Integr Biol. 2012;16:284-7.

24. Cameron ND, Enser MB. Fatty acid composition of lipid in Longissimus dorsi muscle of Duroc and British Landrace pigs and its relationship with eating quality. Meat Sci. 1991;29:295-307.

25. Fontanesi L, Schiavo G, Galimberti G, Bovo S, Russo V, Gallo M, et al. A genome-wide association study for a proxy of intermuscular fat level in the Italian Large White breed identifies genomic regions affecting an important quality parameter for dry-cured hams. Anim Genet. 2017;48:459-65. 
26. Chen M, Wang J, Wang Y, Wu Y, Fu J, Liu J. Genome-wide detection of selection signatures in Chinese indigenous Laiwu pigs revealed candidate genes regulating fat deposition in muscle. BMC Genet. 2018;19:31.

27. Piórkowska K, Małopolska M, Ropka-Molik K, Szyndler-Nędza M, Wiechniak A, Żukowski K, et al. Evaluation of SCD, ACACA and FASN Mutations: Effects on Pork Quality and Other Production Traits in Pigs Selected Based on RNA-Seq Results. Animals. 2020;10:123.

28. Lee J-B, Kang Y-J, Kim S-G, Woo J-H, Shin M-C, Park N-G, et al. GWAS and Post-GWAS High-Resolution Mapping Analyses Identify Strong Novel Candidate Genes Influencing the Fatty Acid Composition of the Longissimus dorsi Muscle in Pigs. Genes (Basel). 2021;12:1323.

29. Jin P, Wu X, Xu S, Zhang H, Li Y, Cao Z, et al. Differential expression of six genes and correlation with fatness traits in a unique broiler population. Saudi J Biol Sci. 2017;24:945-9.

30. Chermuła B, Brązert M, Jeseta M, Ożegowska K, Sujka-Kordowska P, Konwerska A, et al. The Unique Mechanisms of Cellular Proliferation, Migration and Apoptosis are Regulated through Oocyte Maturational Development-A Complete Transcriptomic and Histochemical Study. Int J Mol Sci. 2018;20:84.

31. Gao N, Chen Y, Liu X, Zhao Y, Zhu L, Liu A, et al. Weighted single-step GWAS identified candidate genes associated with semen traits in a Duroc boar population. BMC Genomics. 2019;20:797.

32. Ramayo-Caldas Y, Fortes MRS, Hudson NJ, Porto-Neto LR, Bolormaa S, Barendse W, et al. A marker-derived gene network reveals the regulatory role of PPARGC1A, HNF4G, and FOXP3 in intramuscular fat deposition of beef cattle. J Anim Sci. 2014;92:2832-45.

33. Si Z, Guan X, Teng X, Peng X, Wan Z, Li Q, et al. Identification of CYP46A1 as a new regulator of lipid metabolism through CRISPR-based whole-genome screening. FASEB J. 2020;34:13776-91.

34. Szabó A, Xiao X, Haughney M, Spector A, Sahin-Tóth M, Lowe ME. A novel mutation in PNLIP causes pancreatic triglyceride lipase deficiency through protein misfolding. Biochim Biophys Acta - Mol Basis Dis. 2015;1852:1372-9.

35. Zhou X, Guo W, Yin H, Chen J, Ma L, Yang Q, et al. Whole Exome Sequencing Study in a Family with Type 2 Diabetes Mellitus. Int J Gen Med. 2021;Volume 14:8217-29.

36. Fu Y, Fu J, Wang A. Association of EphA4 polymorphism with swine reproductive traits and mRNA expression of EphA4 during embryo implantation. Mol Biol Rep. 2012;39:2689-96.

37. Edea Z, Hong J-K, Jung J-H, Kim D-W, Kim Y-M, Kim E-S, et al. Detecting selection signatures between Duroc and Duroc synthetic pig populations using high-density SNP chip. Anim Genet. 2017;48:473-7.

38. Tan C, Wu Z, Ren J, Huang Z, Liu D, He X, et al. Genome-wide association study and accuracy of genomic prediction for teat number in Duroc pigs using genotyping-by-sequencing. Genet Sel Evol. 2017;49:35.

39. Nishizuka M, Hayashi T, Asano M, Osada S, Imagawa M. KCNK10, a Tandem Pore Domain Potassium Channel, Is a Regulator of Mitotic Clonal Expansion during the Early Stage of Adipocyte Differentiation. Int J Mol Sci. 2014;15:22743-56.

40. Schiavo G, Bertolini F, Utzeri VJ, Ribani A, Geraci C, Santoro L, et al. Taking advantage from phenotype variability in a local animal genetic resource: identification of genomic regions associated with the hairless phenotype in Casertana pigs. Anim Genet. 2018;49:321-5. 
41. Bai C, Pan Y, Wang D, Cai F, Yan S, Zhao Z, et al. Genome-wide association analysis of residual feed intake in Junmu No. 1 White pigs. Anim Genet. 2017;48:686-90.

42. Cesar ASM, Regitano LCA, Koltes JE, Fritz-Waters ER, Lanna DPD, Gasparin G, et al. Putative Regulatory Factors Associated with Intramuscular Fat Content. Davoli R, editor. PLoS One. 2015;10:e0128350.

43. Li X, Yao X, Xie H, Deng M, Gao X, Deng K, et al. Effects of SPATA6 on proliferation, apoptosis and steroidogenesis of Hu sheep Leydig cells in vitro. Theriogenology. 2021;166:9-20.

44. Yuan S, Stratton CJ, Bao J, Zheng H, Bhetwal BP, Yanagimachi R, et al. Spata6 is required for normal assembly of the sperm connecting piece and tight head-tail conjunction. Proc Natl Acad Sci. 2015;112:E430-9.

\section{Tables}

Table 1. Tissue-specific genes that overlapped with the genome-wide significant GPSM signals 


\begin{tabular}{|c|c|c|c|c|c|c|}
\hline $\mathrm{Chr}^{1}$ & Position & SNP & Gene $^{2}$ & Description & Tissue $^{3}$ & $\begin{array}{l}\text { Type of } \\
\text { orthologue }\end{array}$ \\
\hline 1 & 68477634 & rs343429147 & GRIK2 & $\begin{array}{l}\text { Glutamate ionotropic } \\
\text { receptor kainate type } \\
\text { subunit } 2\end{array}$ & Cerebellum & 1 -to-1* \\
\hline 5 & 17275197 & rs325589813 & ACVRL 1 & $\begin{array}{l}\text { Activin A receptor like } \\
\text { type } 1\end{array}$ & Lung & 1 -to-1* \\
\hline 6 & 44902082 & rs333577047 & FFAR2 & $\begin{array}{l}\text { Free fatty acid receptor } \\
2\end{array}$ & Spleen & 1 -to-1* \\
\hline 6 & 45249022 & 6_45249022 & NPHS1 & $\begin{array}{l}\text { NPHS1 adhesion } \\
\text { molecule, nephrin }\end{array}$ & Kidney & 1 -to-1* \\
\hline 8 & 4497067 & rs325024132 & JAKMIP1 & $\begin{array}{l}\text { Janus kinase and } \\
\text { microtubule interacting } \\
\text { protein } 1\end{array}$ & Cerebrum & 1 -to-1* \\
\hline 8 & 126046420 & rs325782763 & GRID2 & $\begin{array}{l}\text { Glutamate ionotropic } \\
\text { receptor delta type } \\
\text { subunit } 2\end{array}$ & Cerebellum & 1 -to-1* \\
\hline 12 & 921691 & rs321539153 & FASN & Fatty acid synthase & Fat & 1 -to-1 \\
\hline 12 & 1136822 & 12_1136822 & PPP1R27 & $\begin{array}{l}\text { Protein phosphatase } 1 \\
\text { regulatory subunit } 27\end{array}$ & Muscle & 1 -to-1* \\
\hline 12 & 44658312 & rs694026589 & $V T N$ & Vitronectin & Liver & 1-to-many* \\
\hline 12 & 44734028 & rs328927732 & $S L C 13 A 2$ & $\begin{array}{l}\text { Solute carrier family } 13 \\
\text { member } 2\end{array}$ & Duodenum & 1 -to-1* \\
\hline 12 & 54906956 & rs329254243 & GAS7 & Growth arrest specific 7 & Cerebrum & 1 -to-1* \\
\hline 15 & 17161987 & rs322661315 & $A C M S D$ & $\begin{array}{l}\text { Aminocarboxymuconate } \\
\text { semialdehyde } \\
\text { decarboxylase }\end{array}$ & Kidney & 1 -to-1* \\
\hline 16 & 27537893 & rs335570719 & SELENOP & Selenoprotein P & Liver & 1 -to-1* \\
\hline 17 & 2034960 & 17_2034960 & $S G C Z$ & Sarcoglycan zeta & Cerebrum & 1 -to-1* \\
\hline 17 & 9824048 & rs331351838 & ZMAT4 & Zinc finger matrin-type 4 & Cerebrum & 1 -to-1* \\
\hline
\end{tabular}

${ }^{1}$ Sus scrofa chromosome. ${ }^{2}$ The promising candidate genes. ${ }^{3}$ Specific expression tissues. ${ }^{4}$ A type of orthologue assigned for sus scrofa and homo sapiens.

Table 2. Tissue-specific genes that overlapped with teat number relevant traits-specific selection signature 


\begin{tabular}{|c|c|c|c|c|c|c|}
\hline $\mathrm{Chr}^{1}$ & Start ${ }^{2}$ & End3 & Gene $^{4}$ & Description & Tissue $^{5}$ & $\begin{array}{l}\text { Type of } \\
\text { orthologue }\end{array}$ \\
\hline 4 & 71960001 & 72010000 & ASPH & $\begin{array}{l}\text { Aspartate beta- } \\
\text { hydroxylase }\end{array}$ & Fat & 1 -to-1* \\
\hline 7 & 120550001 & 120600000 & CCDC85C & $\begin{array}{l}\text { Coiled-coil domain } \\
\text { containing } 85 \mathrm{C}\end{array}$ & Cerebellum & 1 -to-1* \\
\hline 7 & 120650001 & 120700000 & CYP46A1 & $\begin{array}{l}\text { Cytochrome P450 family } \\
46 \text { subfamily A member } \\
1\end{array}$ & Cerebrum & 1 -to-1* \\
\hline 12 & 52430001 & 52480000 & ASGR2 & $\begin{array}{l}\text { Asialoglycoprotein } \\
\text { receptor } 2\end{array}$ & Liver & 1-to-1* \\
\hline 14 & 126740001 & 126820000 & PNLIP & Pancreatic lipase & Duodenum & 1 -to-1* \\
\hline 14 & 126740001 & 126820000 & PNLIPRP1 & $\begin{array}{l}\text { Pancreatic lipase related } \\
\text { protein } 1\end{array}$ & Duodenum & 1 -to-1* \\
\hline 15 & 20730001 & 20780000 & DPP10 & $\begin{array}{l}\text { Dipeptidyl peptidase like } \\
10\end{array}$ & Cerebrum & 1 -to-1* \\
\hline 15 & 123440001 & 123490000 & EPHA4 & EPH receptor A4 & Cerebrum & 1-to-1* \\
\hline 17 & 18010001 & 18060000 & PLCB4 & Phospholipase $\mathrm{C}$ beta 4 & Cerebellum & 1 -to-1* \\
\hline
\end{tabular}

${ }^{1}$ Sus scrofa chromosome. ${ }^{2}$ The start of the potential selection regions. ${ }^{3}$ The end of the potential selection regions. ${ }^{4}$ The promising candidate genes. ${ }^{5}$ Specific expression tissues. ${ }^{6} \mathrm{~A}$ type of orthologue assigned for sus scrofa and homo sapiens.

\section{Figures}


A

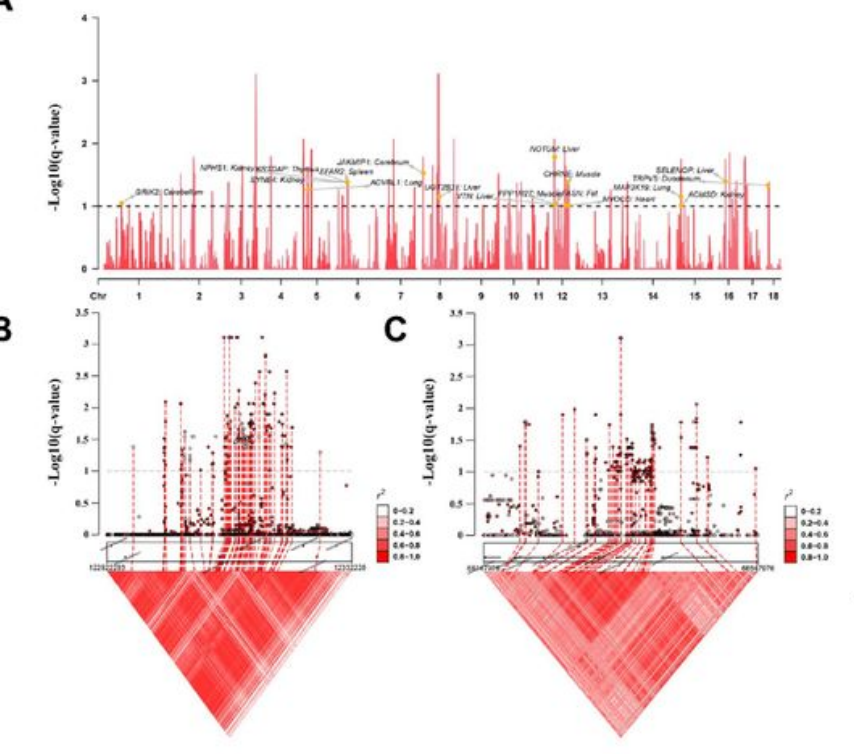

D

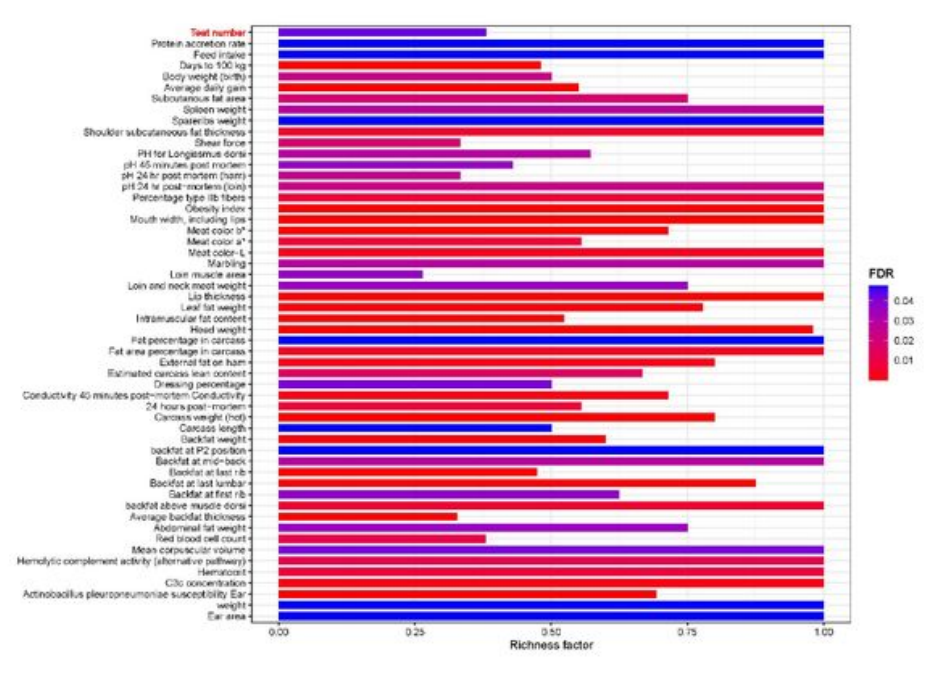

\section{Figure 1}

GPSM detects signatures of ongoing polygenic selection in the Chinese Duroc pigs. (A) GPSM Manhattan plots for the Chinese Duroc pigs. The Y-axis displayed the -log10(q-value) of SNP according to their chromosomal position (X-axis). The horizontal dashed line depicts the genome-wide significance level ( $q$-value $<0.1$ ). The significant variants were annotated using tissue-specific genes. (B) The LD block in significant chromosome regions lacated in Sus scrofa chromosome (SSC) 3. (C) The LD block in significant chromosome regions lacated in SSC 8. (D) QTL enrichment analyses with GPSM signals. The richness factor was obtained by the ratio of the number of QTLs annotated in the candidate regions and the total number of each QTL 


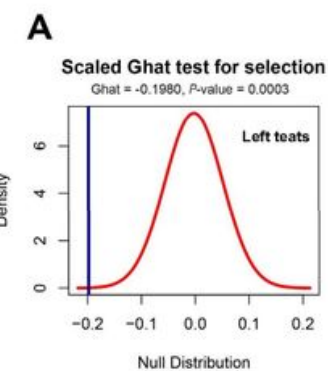

C

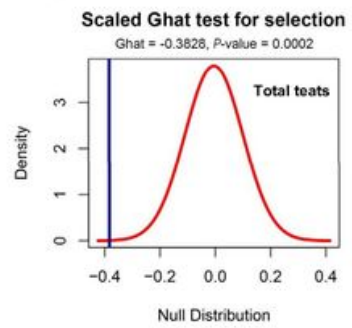

B

Scaled Ghat test for selection

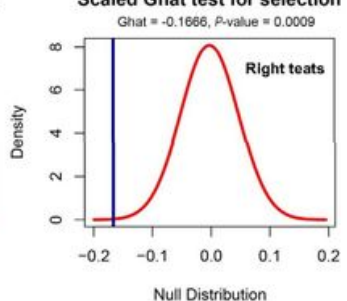

D

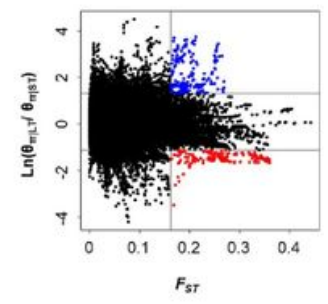

E

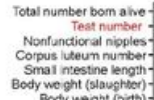

Avergege daty gai
tencerness scose

ubcutanous tat are

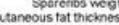

Shoulder external fat weght
Shear force at fist peak
Shear force

PH lor Senimembranosis

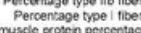

Meat molor soo

Neat colock
N muscio are

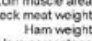

Ham pescentag

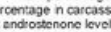

at-cits pesconta

Dresty fipid contion

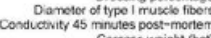

Carcass weight (1)
Carcess weight 100

Carcass lengit

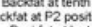

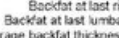

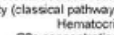

Csc concentritation
.

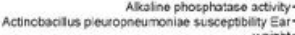

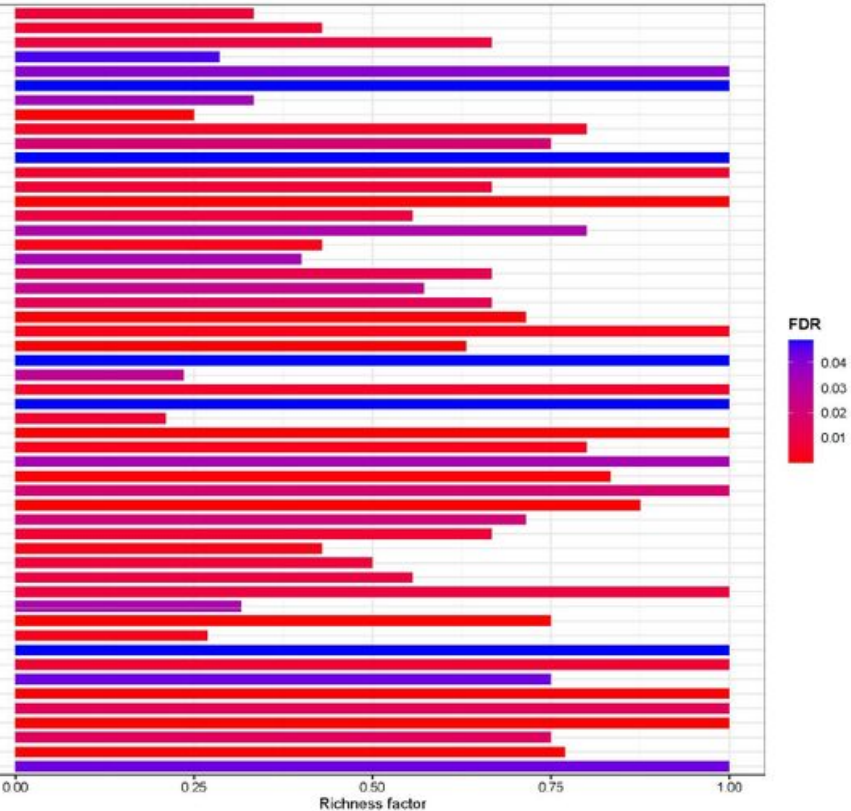

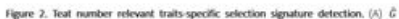

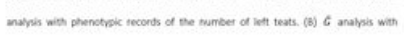

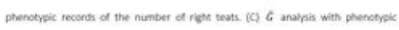

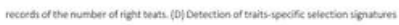

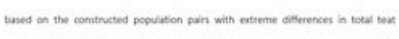

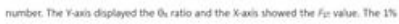

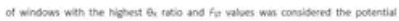

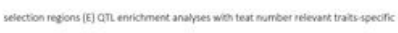

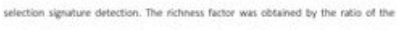

Figure 2

See image above for figure legend. 

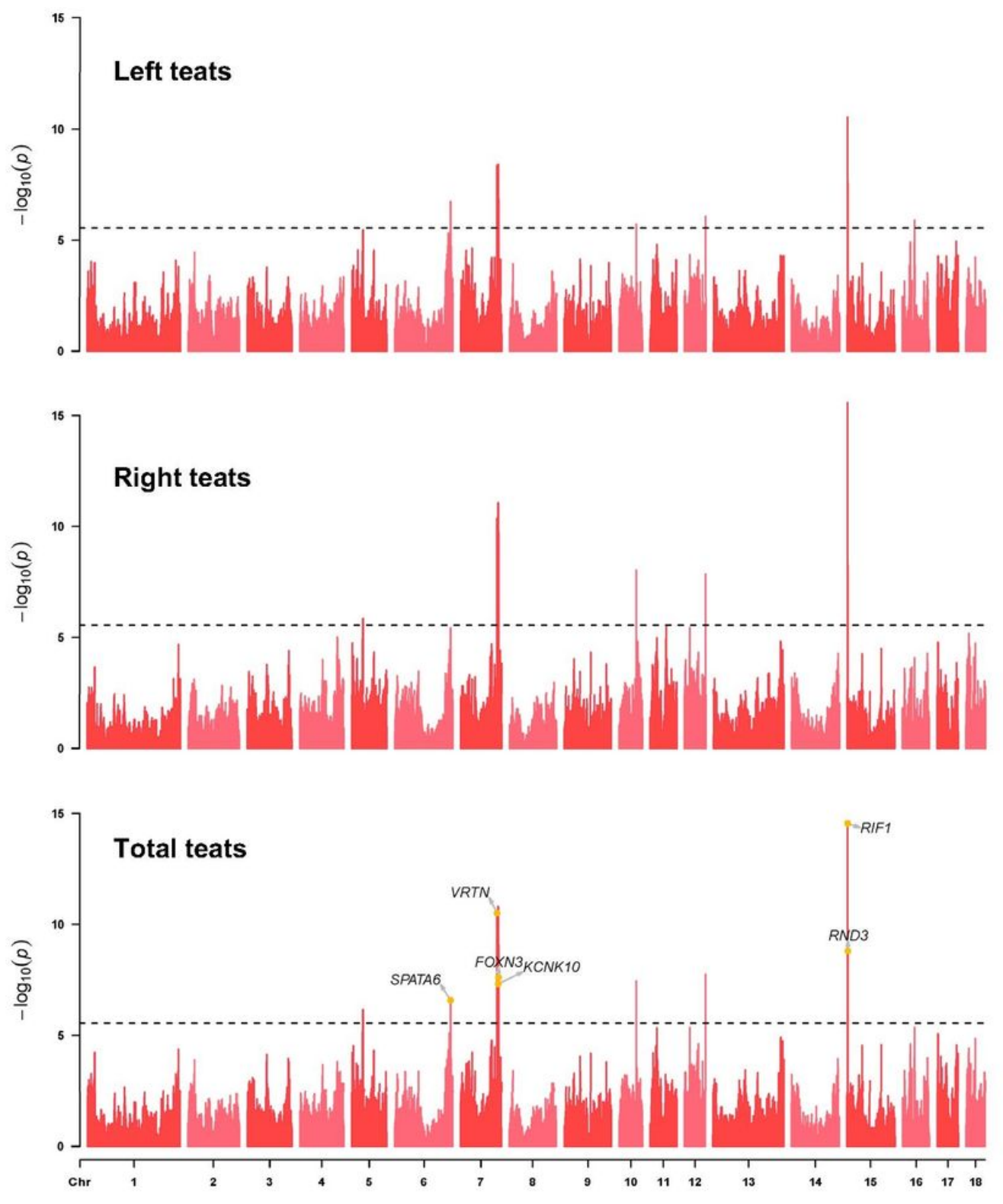

Figure 3

The Manhattan plots of GWAS for teat number relevant traits. The Y-axis of Manhattan plots displayed the $\log 10$ (P-values) of each SNP in the genome wide association analysis, the $X$-axis represented the position of SNPs for chromosomes. 

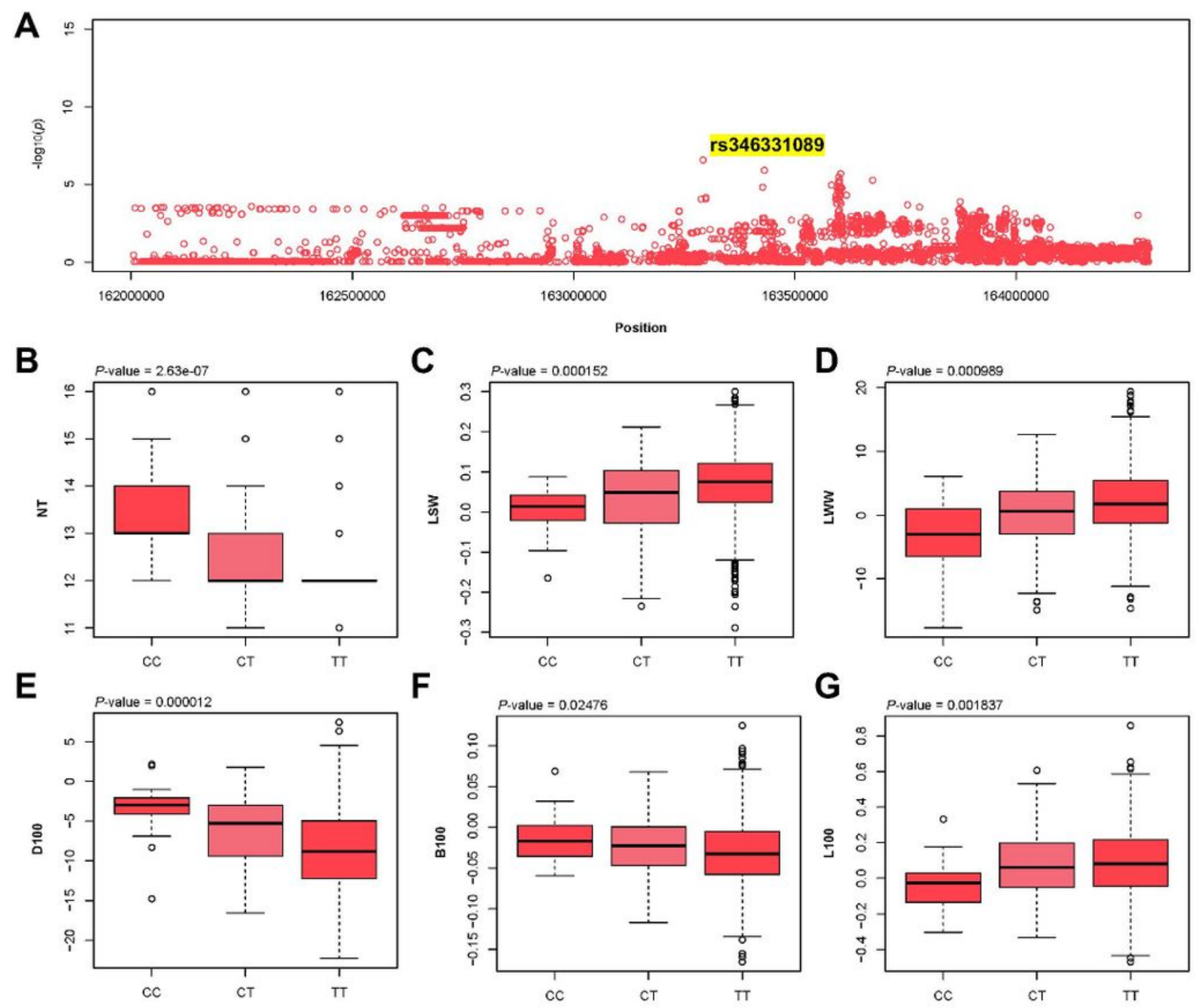

Figure 4

The pleiotropic variant rs346331089 and its effects on economic traits. (A) Regional association plots around rs324534752. Genotype effect plots of rs346331089 among three types for teat number (B), litter size at weaning (C), litter weight at weaning (D), days to $100 \mathrm{~kg}(\mathrm{E})$, backfat thickness at $100 \mathrm{~kg}(\mathrm{~F})$, and loin muscle area at $100 \mathrm{~kg}(\mathrm{G})$.

\section{Supplementary Files}

This is a list of supplementary files associated with this preprint. Click to download.

- AdditionalTables.xlsx

- Additionalfigures.docx 